\title{
Static Soil Moisture Retrieval Measurements Based on GNSS-R
}

\author{
TENGDA Pei ${ }^{1, a}$ and YUEKUN Pei ${ }^{1, b}$ \\ ${ }^{1}$ Street Xuefudajie, 10, Dalian Economic Technological Development Zone, Liaoning, China, \\ 116622 \\ a757103563@qq.com, bpeiyuekun@126.com
}

Keywords: GNSS-R, Soil moisture, Software receiver, Dielectric constant.

\begin{abstract}
GNSS Reflectometry (GNSS-R) is a remote sensing tool for extracting Earth surface information. This work presents a preliminary result of a static measurement applying the software GPS receiver. The system configuration consists of both Right Hand Circularly Polarization (RHCP) and Left Hand Circularly Polarization (LHCP) antennas for receiving reflected signals. Raw sampled data were stored into two PCs for post processing in order to get Signal to Noise Ratio (SNR) for both polarizations. Power reflectivity behavior was theoretically analyzed and compared with obtained results. A trial dielectric constant retrieval of the meadow was attempted, and the results were coherent with the in-situ soil moisture state.
\end{abstract}

\section{Introduction}

Global Navigation Satellite System Reflectometry (GNSS-R) has been widely studied for remote sensing of the Earth surfaces such as sea wind and roughness, snow and ice depth, vegetation coverage and soil moisture since it was first brought out in 1993 [1]. GNSS transmits signals lying in $\mathrm{L}$ band, therefore the received reflected signal is sensitive enough to the relative permittivity of the terrain (top 0-4cm). More recently, soil dielectric constant and soil moisture retrieval has started to produce some results. Three different observing strategies were implemented for this kind of applications: (1) Multipath effect and it's relation to soil moisture [2]. It exploits the relationship between the total received signal amplitude behavior and the soil moisture for the retrieval. (2) Interference Pattern Technique (IPT). Soil moisture retrieval is based on finding a specific 'notch' point from the interference pattern, versus satellite elevation [3]. (3) Bi-static method. This is based on the separate reception of direct and reflected signals using different antennas and on the separate measurement of signal powers. Depending on the antenna configuration, two possible observing systems exploiting the bi-static geometry can be further identified: (a) A down-looking Left Hand Circularly Polarization (LHCP) antenna and an up-looking RHCP antenna. LHCP antenna receives reflected GPS signal from the surface. Power reflectivity could be obtained either by using a bi-static radar equation or from the power ratio between the reflected signal and the direct signal [4]. Reflectivity is then a function of dielectric constant of the soil, the elevation angle and the surface 
roughness. (b) One RHCP up-looking antenna and two down-looking antennas with one RHCP polarized and the other LHCP polarized [5]. With this configuration, it's possible to measure both the co-polar component of the terrain reflectivity (using LHCP signal) and the cross-polar component (using the RHCP antenna). The ratio of these two reflectivities was verified to be in good correlation with soil moisture and it was independent from the surface roughness. In this work, the receiving system is as depicted by (b) above and is shown in Fig. 1. It's based on GPS software receiver. The RHCP and LHCP antennas dedicated for receiving reflected signal mounted on an arm are fixed on a tripod. The elevation angle of the arm is adjustable depending on the specular point position on the ground. Raw signals were down converted and sampled into digital values by radio frequency front-ends (SiGE GPS frontend) connected to the antennas. Digital binary data were further restored into laptops separately through data grabber software NGrab developed by NAVSAS Group of Politecnico di Torino, Italy. A sampling frequency of $8.1838 \mathrm{MHz}$ is used, giving about 8 samples per $\mathrm{C} / \mathrm{A}$ code chip. For static measurements, no navigation information is required from direct signal, but direct signal can be used as reference for calculating power reflectivity. However, our setup doesn't consist of RHCP antenna pointing upward to receive direct signal since our first concern is to derive dielectric constant of soil through the ratio between co- and cross-polarization reflectivities in which case the direct signal contribution can be canceled out. See details in the following section. But it's easy to add the direct signal receiving part depending on the retrieval process we decide to use.

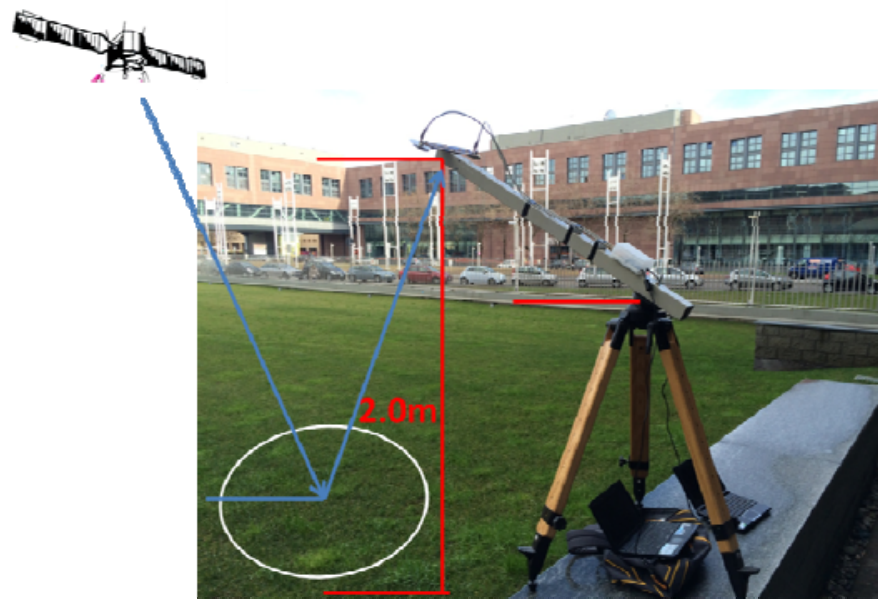

Fig. 1 Receiver set up and geometry, the white ellipse indicates the Fresnel Zone

The total raw-sampled reflected GNSS signals acquired during the measurement were processed into Delay Doppler Maps (DDMs) and Delay Waveforms exploiting a fully open loop scheme, in order to evaluate Signal to Noise Ratios (SNRs) time series, without the necessity to wait for standard GNSS close loop acquisition and tracking. For each available satellite, and with a 0.5 second interval, a SNR value related to the received signal is recorded. As shown in Fig. 1, we did several experiments on the meadow in front of the GM building of Turin. The time slot was carefully chosen by using our specular point prediction software such that the specular points of two GPS satellites were close enough for comparison of the retrieved results. One of the dielectric constant retrieval result of the meadow is shown in the result section and it coincides with the in-situ soil moisture.

\section{Retrieval Method}

Starting from SNR of direct RHCP signal and reflected LHCP and RHCP signals, the retrieval process is shown in the following: 


$$
S N R_{L}^{r}=\frac{P_{L}^{r}}{P_{N}^{r}}, S N R_{R}^{r}=\frac{P_{R}^{r}}{P_{N}^{r}}, S N R_{R}^{d}=\frac{P_{R}^{d}}{P_{N}^{d}} .
$$

where $P$ is signal power, superscripts $r$ and $d$ states for reflected and direct path, subscripts $L, R$ and $N$ are LHCP, RHCP and Noise, respectively. Power reflectivity characterizing the surface received by LHCP and RHCP antennas are proportional to:

$$
R_{L R} \propto \frac{P_{L}^{r}}{P_{R}^{d}}, R_{R R} \propto \frac{P_{R}^{r}}{P_{R}^{d}} .
$$

If the surface is perfectly smooth, the power reflectivity of specular reflection can be written as the modulus square of Fresnel reflection coefficient without regard to the surface roughness by the criteria described in [6],

$$
R_{L R}=\left|\Gamma_{L R}(\theta, \varepsilon)\right|^{2}, R_{R R}=\left|\Gamma_{R R}(\theta, \varepsilon)\right|^{2} .
$$

where $\Gamma$ is Fresnel coefficient, $\vartheta$ is the incidence angle of GPS signal (complementary angle of GPS satellite elevation), $\varepsilon$ is relative dielectric constant of the soil. Furthermore, $\varepsilon$ is proportional to soil moisture for a certain soil type. Applying the Fresnel reflection coefficient equations [7], the relationships between $\left|\Gamma_{L R}\right|\left(\left|\Gamma_{R R}\right|\right)$ and elevation angle of satellite under 3 different soil moisture conditions (for sand) are shown in Fig. 2:

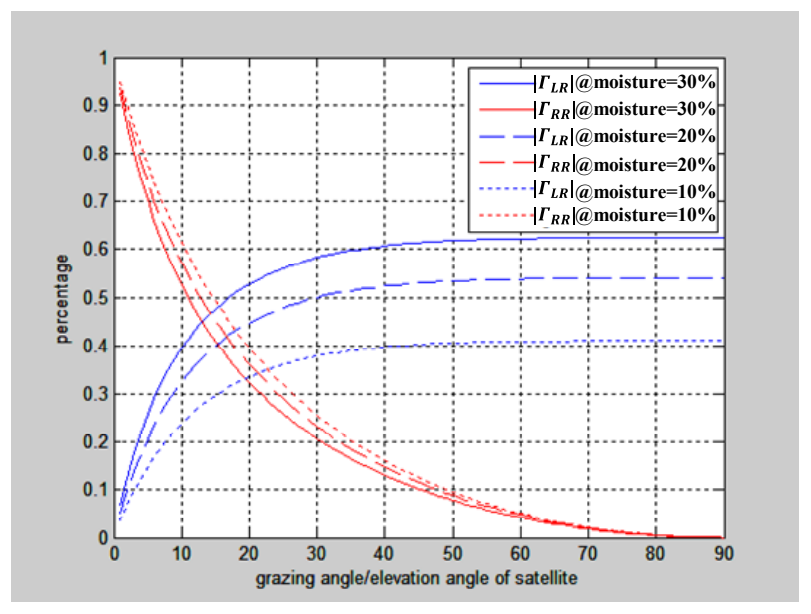

Fig. 2 Relationships between $\left|\Gamma_{L R}\right|\left(\left|\Gamma_{R R}\right|\right)$ and elevation angle of satellite under 3 different soil moisture conditions

As shown in Fig. 2, the LHCP Fresnel coefficient $\left|\Gamma_{L R}\right|$ is much higher than $\left|\Gamma_{R R}\right|$ when the satellite elevation angle is high. $\left|\Gamma_{L R}\right|$ increases while $\left|\Gamma_{R R}\right|$ decreases as the volumetric soil moisture increases. As our measurements were planned to receive signals of satellite with higher elevation angle, and it could be seen from Fig. 2 that $\left|\Gamma_{L R}\right|$ should be higher than $\left|\Gamma_{R R}\right|$. Regarding Eq. 1, 2 and 3, we expect $S N R_{L}^{r}$ higher than $S N R_{R}^{r}$ in a perfectly smooth surface condition, and combine the equations we could derive dielectric constant $\varepsilon$ from SNR computed.

\section{Measurements and Results}

First trial experiment was done using the geometry and configuration as shown in Fig. 1, two rays of signal were expected to have signal reflections from the meadow during the data acquisition, which are PRN 26 and PRN 28. Results of SNR of both RHCP and LHCP during the one minute data collection were shown in Fig. 3. 


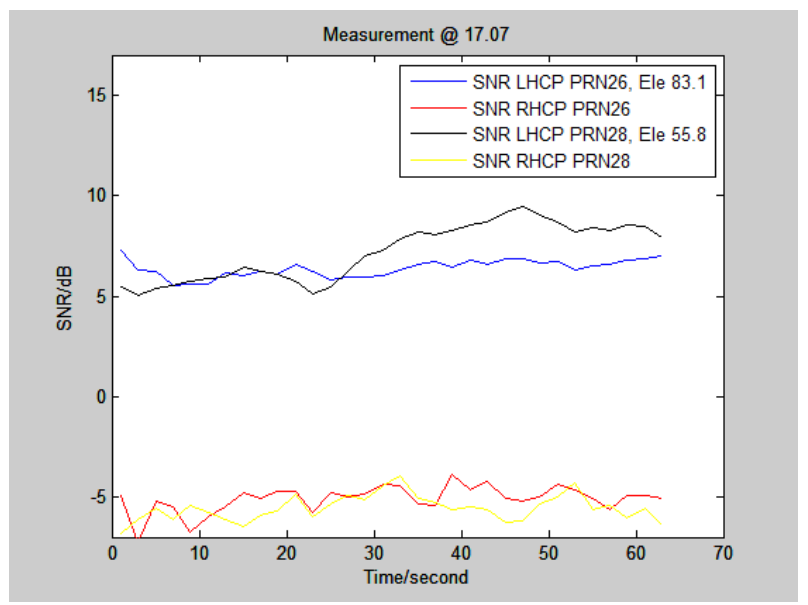

Fig. 3 SNR measurements on both RHCP and LHCP of PRN 26 and PRN 28

As shown in Fig. 3, for both PRN 26 and PRN 28, SNR of LHCP is more than 10dB higher than that of RHCP. During time, SNRs with same polarization are in the similar level, except that the LHCP SNR from PRN 28 has about $3 \mathrm{~dB}$ increase. This could be resulted from several reasons including transmitted power attenuation, surface roughness or grass coverage.

Applying our retrieval algorithm, the preliminary results of dielectric constant obtained from SNR of PRN28 is shown in Fig. 4. The trend of the dielectric constant is similar with and mainly determined by the LHCP SNR, comparing with the black line in Fig. 3. The retrieved dielectric constant was relatively low and it corresponded to the in-situ soil moisture state, which was very dry.

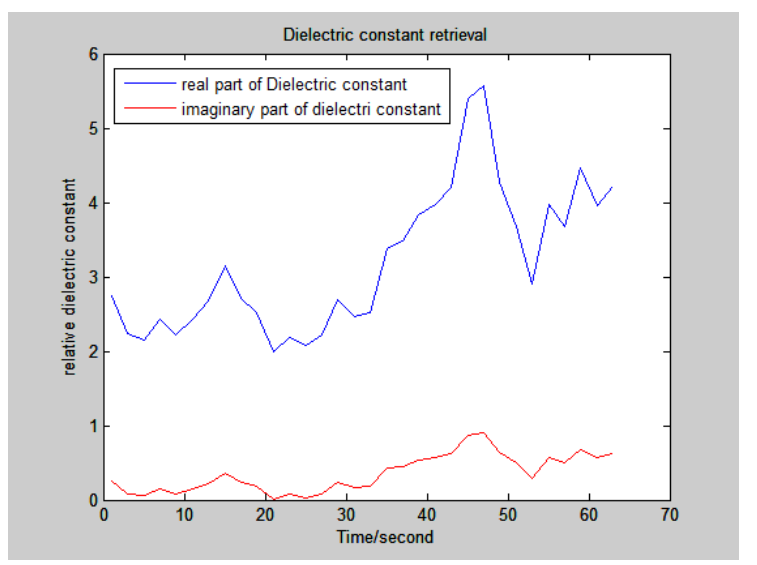

Fig. 4 Retrieved dielectric constant from the output SNR of PRN 28 shown in Fig. 3

\section{Conclusions}

The configuration using both LHCP and RHCP to receive reflected GPS signals were applied and tested. Preliminary results show that the SNR follows the principle of LHCP and RHCP reflectivity. A further RHCP antenna for receiving direct signal as reference can be exploited in order to eliminate the effect of direct signal power change and to get power reflectivity directly. Dielectric constant retrieval processes are under studying and estimating considering the surface roughness state and other influence.

Future work is on getting a more reliable dielectric constant retrieving algorithm and its verification. Further measurement campaigns are under planning, with the remote sensed surface well 
known and controlled (soil moisture content can be manually changed and monitored), and the retrieved dielectric constant will be compared with the in-situ measurement.

\section{References}

[1] S. Jin, G. P. Feng, and S. Gleason, "Remote sensing using GNSS signals: Current status and future directions," Advances in Space Research, vol. 47, pp.1645-1653. (2011)

[2] K. M. Larson, J. J. Braun, E. E. Small, V. U. Zavorotny,E. D. Gutmann, and A. L. Bilich, "GPS multipath and its relation to near-surface soil moisture content," IEEE Journal of Selected Topics in Applied Earth Observations and Remote Sensing, vol. 3, no. 1, pp. 91-99. (2010)

[3] N. Rodriguez-Alvarez, X. Bosch-Lluis, A. Camps, A. Aguasca, M. Vall-Llossera, E. Valencia, I. Ramos-Perez, and H. Park, "Review of crop growth and soil moisture monitoring from a ground-based instrument implementing the Interference Pattern GNSS-R technique," Radio Science, vol. 46, RS0C03, pp. 1-11. (2011)

[4] S. Gleason, S. Hodgart, Y. Sun, C. Gommenginger, S. Mackin, M. Adjrad, and M. Unwin, "Detection and processing of bistatically reflected GPS signals from low earth orbit for the purpose of ocean remote sensing,", IEEE Transactions on Geoscience and Remote Sensing, vol. 43, no. 6, pp. 1229-1241. (2005)

[5] A. Egido, M. Caparrini, G. Ruffini, S. Paloscia, E. Santi,L. Guerriero, N. Pierdicca and N. Floury, "Global navigation satellite systems reflectometry as a remote sensing tool for agriculture," Remote Sensing, no. 4, pp. 2356-2372. (2012)

[6] F. T. Ulaby, R. K. Moore, and A. K. Fung, "Microwave remote sensing: active and passive", Addison Wesley, vol. 3. (1986)

[7] S. J. Katzberg and J. L. Garrison Jr. "Utilizing GPS to determine ionospheric delay over the ocean," National Aeronautics and Space Administration, Langley Research Center. (1996) 